\title{
A two-axis cable-driven ankle-foot mechanism
}

\author{
Evandro M Ficanha ${ }^{1}$, Mohammad Rastgaar ${ }^{1 *}$ and Kenton R Kaufman ${ }^{2}$
}

\begin{abstract}
This paper describes a novel cable-driven ankle-foot mechanism with two controllable degrees of freedom (DOF) in dorsiflexion-plantarflexion (DP) and inversion-eversion (IE). The presented mechanism is a proof of concept to demonstrate feasibility. Ankle kinematic measurements demonstrate that ankle IE rotations during a step turn are significantly different from walking on a straight path. This suggests that the ankle-foot mechanisms used in prostheses, exoskeletons, and bipedal robots can be improved by controlling a second degree of freedom in the frontal plane. The proposed prototype mechanism is described in detail, and its design considerations and parameters are presented. The mechanism is capable of producing trajectories similar to the human ankle during a step turn. The device shows passive mechanical impedance close to the human ankle mechanical impedance, allowing its mechanical impedance to be controlled using an impedance controller. The presented mechanism is capable of providing key mechanical characteristics similar to the human ankle, including power, range of motion, and weight, suggesting the feasibility of this design concept.
\end{abstract}

Keywords: Ankle mechanism; Ankle impedance; Two-DOF ankle-foot; Turning; Ankle rotations; Cable-driven prosthesis; Human ankle kinematics; Multi-axis ankle-foot prosthesis; Powered lower extremity prosthesis

\section{Background}

Cable-driven mechanisms have been used on upper extremity exoskeletons and assistive robots such as prosthetic hands. In such mechanisms, pulling the cables results in motion, the same way that the muscles pull tendons in the musculoskeletal system. Exploiting a cable-driven system allows the DC motors to be placed at locations that are not necessarily close to the mechanisms' joints, hence, assisting in maintaining a low profile and reducing the size and weight of the device. One example of such design is lower extremity powered exoskeleton (LOPES) with eight actuated degrees of freedom at the hip, knee, and pelvis [1].

A similar cable-driven design concept can be followed in the lower extremity prostheses design to extend the controllable degrees of freedom (DOFs) beyond state of the art. Currently available ankle-foot prostheses are capable of controlling the ankle joint in only a single DOF in the sagittal plane. These prostheses improve the quality of life of amputees and are designed to increase mobility. However, turning plays a major role in activities of daily living

\footnotetext{
* Correspondence: rastgaar@mtu.edu

${ }^{1}$ Department of Mechanical Engineering-Engineering Mechanics, Michigan

Technological University, Houghton, Michigan 49931, USA

Full list of author information is available at the end of the article
}

and account for an average of $25 \%$ of steps, ranging from $8 \%$ to $50 \%$ of all steps depending on the activity [2]. Turning requires modulation of ankle impedance in the sagittal and frontal planes to control lateral and propulsive ground reaction forces in order to accelerate the body center of mass along the gait path, resulting in increased lateral and propulsive impulses when compared to a straight step [3]. As a result, turning strategies for amputees and nonamputees are different. Non-amputees rely on hip movement in the frontal plane and moment generation at the ankle joint, while amputees using passive prostheses rely on hip extension in the sagittal plane to compensate for the lack of forward propulsion from their passive prosthesis [4-7]. This data suggests that the next generation of ankle-foot prosthesis can benefit from designs that are capable of functioning in both the frontal and sagittal planes. A cable-driven mechanism seems to be a suitable solution to overcome the weight, size, and profile constraints while introducing a second actuation component. An ankle-foot mechanism capable of impedance modulation and power generation in both the sagittal and fontal planes allows the user to turn in arbitrary directions and walk on slopes that will increase their agility. 
When physical systems interact with each other, they behave either as an impedance (i.e., accepts external motion inputs and generates force outputs) or an admittance (i.e., accepts external force inputs and generates motion outputs) [8]. The human ankle functions by adjusting its mechanical impedance and capability of producing net positive work during push-off. These capabilities have inspired the design of several new anklefoot prostheses. One example is a transfemoral prosthesis with powered ankle and knee joints in the sagittal plane developed by Sup et al. where the controller is capable of regulating the impedance of each joint as needed [9-12]. SPARKy 1 and 2 have been developed by Hitt et al. as a tendon-actuated ankle-foot prostheses capable of producing the necessary push-off moments during walking and running, respectively [13,14]. Additionally, $\mathrm{Au}$ et al. developed the BiOM, an ankle-foot prosthesis with a finite state machine to determine the gait phase and generate the appropriate kinetic output [15-19]. All three of the aforementioned devices are capable of increasing mobility in the sagittal plane by producing net positive work in the sagittal plane. Finally, Bellman et al. conceived SPARKy 3, an ankle robot with two DOFs; however, a prototype design has not been developed [20]. This leads to the fact that a design to address the weight and size limitations is necessary to develop the next generation of ankle-foot prostheses capable of properly functioning in both the sagittal and frontal planes. These additional capabilities will allow a more natural gait, especially during turning and traversing inclined planes.

The paper is structured as follows: first, the mechanism design is described in details. Next, a trajectory control and instrumentation necessary for a torque feedback is described. Finally, the evaluation experiments are described showing the mechanism capability for reproducing the human ankle kinematics during a step turn. Additionally, a comparison of the passive mechanical impedance of the mechanism's ankle with the human ankle is provided.

\section{Methods}

A prototype cable-driven ankle mechanism with two controllable DOFs in DP and IE directions was designed and fabricated as a proof of concept prototype (Figure 1). The design goal was to demonstrate the feasibility of achieving angular displacements, angular velocities, and torques similar to the human ankle during straight walking and turning in both the sagittal and frontal planes.

\section{Ankle kinematics and design considerations}

A set of gait experiments were performed to compare the ankle kinematics during a step turn and a straight walking step. These experiments were performed to

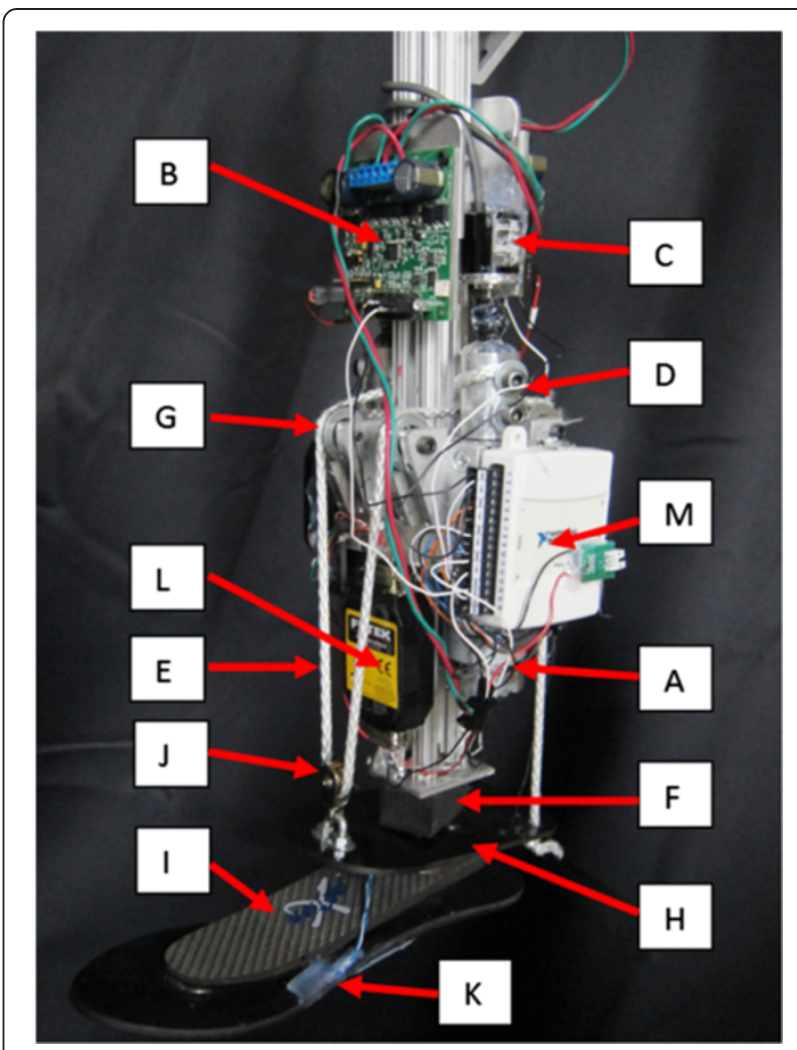

Figure 1 Two-DOF ankle-foot mechanism prototype.

develop preliminary design parameters for the ankle angular displacements and speeds and eventually for evaluation of the prototype mechanism capabilities. A motion capture camera system was used to record the human subjects' ankle kinematics during the stance phase of the gait. The ankle motions during a step turn and straight walking were measured using five male subjects with no reported musculoskeletal disorders. The step turn consisted of pivoting around the leading leg and turning into a new direction approximately perpendicular to the initial direction similar to turning around a corner (Figure 2). Tests were performed at two different speeds to evaluate the effects of the gait speed. A slow speed was set as the preferred speed of the participant for the gait (average of 96 steps per minute); a fast speed was set as the fastest speed the subject felt comfortable to perform the step turn without running (average of 114 steps per minute). The participants provided written informed consent, as approved by the Michigan Technological University Institutional Review Board, prior to their participation in the study.

Ankle kinematics in the frontal plane for the right ankle during the straight steps (Figure 2A) in both slow and fast speeds did not differ; however, during a step turn (Figure 2B), there was a significant increase in the ankle inversion. At straight step and low speed, the ankle 


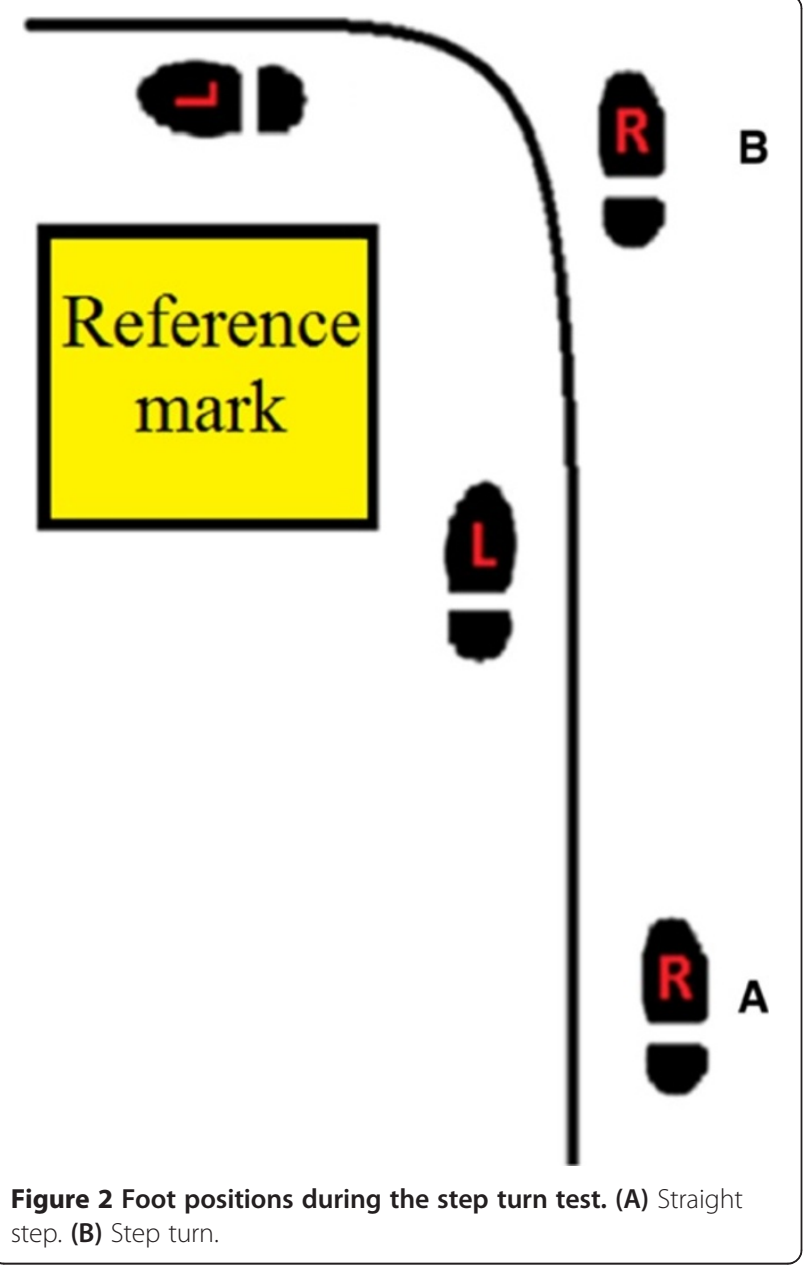

was in $2.8^{\circ}$ eversion during terminal stance, while during the step turn, it changed to $10.5^{\circ}$ inversion. By increasing the speed, $2.5^{\circ}$ eversion and $14.3^{\circ}$ inversion were observed for straight step and step turn, respectively. The changes were equivalent to $248 \%$ and $303 \%$ of the straight step average range of motion (ROM) in the frontal plane for the slow and fast speed tests $\left(5.37^{\circ} \pm 0.6^{\circ}\right.$ and $5.52^{\circ} \pm 0.8^{\circ}$, respectively). Additionally, the average maximum angular velocities among the participants during the fast speed tests were $120 \%$ in the sagittal plane and $101 \%$ in the frontal plane. The maximum angular displacements were $22^{\circ}$ in plantarflexion and $20^{\circ}$ in inversion. These values are important since an anthropomorphic ankle-foot mechanism should be able to reproduce them. There was also an increased rotation of the ankle in IE during turning. This demonstrated the significance of controllable frontal plane rotations in an ankle-foot mechanism for improved maneuverability.

In the transverse plane, the changes in ankle rotations were not as significant as in the frontal plane. The average ankle rotations during the sidestep cut (Figure 2A) decreased during turn at the terminal stance by $-32 \%$ with respect to the average ROM during the straight walk in the transverse plane. However, hip rotations in the transverse plane also need to be accounted in the relative motion of the body with respect to the foot. Further investigation is required to quantify the contributions of the hip and ankle to the transverse plane moments and angles during locomotion before implementing it in the design of ankle-foot mechanisms.

\section{Prototype design}

The prototype design consists of two DC motors and planetary gear heads (A) powered by two motor controllers (B) connected to two quadrature encoders (C) (Figure 1). Two cable drums (D) transfer the required torque to the ankle through the shock-absorbing nylon rope (E). The rope is securely attached to the cable drums to avoid slippage. A universal joint (F) connects the pylon to the foot and is supported by an elastomer to provide passive stiffness and damping to the ankle. The cable is directed to the foot with pulleys $(\mathrm{G})$. The cable is attached to a carbon fiber plate $(\mathrm{H})$, which is connected to a commercially available prosthetic foot (Otto Bock Axtion ${ }^{\circ}$ ) (I). In the rear side of the carbon fiber plate, the cable is mounted to both sides of the longitudinal axis of the foot. At the front side of the carbon fiber plate, the cable passes through a pulley (J). Torque feedback, which will be explored later, is provided by six strain gauges in the foot $(\mathrm{K})$ using two strain gauge amplifiers (L). An analog to digital converter $(\mathrm{M})$ is connected to a remote computer and is used to acquire the sensors data and provide the motor controllers' inputs.

The mechanism is capable of moving in the sagittal plane (DP) when the motors rotate in opposite directions and in the frontal plane (IE) when the motors rotate in the same direction. Also, any combination of DP and IE can be obtained by combining different amounts of rotations in each motor. The proposed mechanical design with two DOFs uses the fundamental principle that three points are sufficient to define a plane in space. If the plane is constrained from translations and has a fixed point of rotation, by defining the position of those three points, all the rotations of the plane can be achieved. In the proposed design (Figure 3 ), three points ( $\mathrm{A}, \mathrm{B}$, and $\mathrm{C}$ ) can be moved in the $\mathrm{Z}$ direction, and thus generate the rotations about the $X$ - and $Y$-axes that are equivalent to DP and IE, respectively, while the universal joint constrains the ankle from rotating about the $Z$-axis. If the two motors move in opposite direction, for example, the left motor rotates about the negative $Z$-axis and the right motor rotates about the positive $Z$-axis with equal magnitude, the resultant driving forces result in both the cable tensions $\overrightarrow{T_{R F}}$ and $\overrightarrow{T_{L F}}$ to be in the positive $\mathrm{Z}$ axis. This causes point $\mathrm{C}$ to move upwards, while points $\mathrm{A}$ and $\mathrm{B}$ move downwards, resulting in dorsiflexion. 


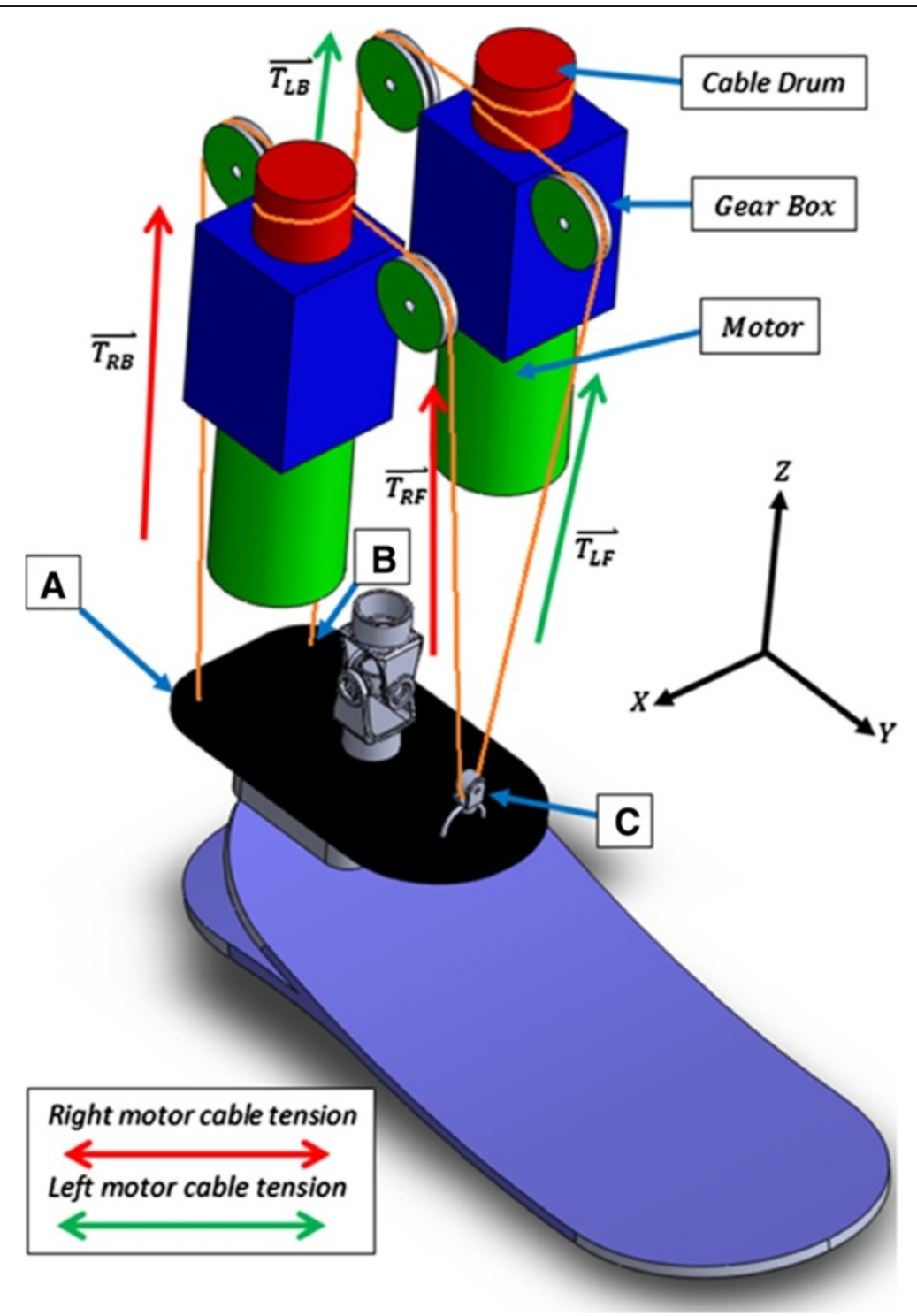

Figure 3 Simplified drawing of the cable-driven mechanism. Points $\mathbf{A}, \mathbf{B}$, and $\mathbf{C}$ are the interaction points between the cable (orange) and the carbon fiber plate. The cable tensions produced by the right motor $\overrightarrow{T_{R F}}$ and $\overrightarrow{T_{R B}}$ for the front and back of the foot respectively, and the cable tensions produced by the left motor $\overrightarrow{T_{L F}}$ and $\overrightarrow{T_{L B}}$ for the front and back of the foot, respectively, are shown. The shin and other components are not shown for simplicity.

If the motors move in the same direction, for example, about the negative $\mathrm{Z}$ axis, the cable tension $\overrightarrow{T_{R B}}$ increases and moves point A upwards; while the cable tension $\overrightarrow{T_{L B}}$ decreases allowing point $\mathrm{B}$ to move downwards. This generates a net eversion torque in the foot. In the front of the foot, since point $\mathrm{C}$ is located on the axis of symmetry of the carbon fiber plate along the $\mathrm{X}$ direction, the amount of cable released by one of cable drum is the same as the cable pulled by the other cable drum. Therefore, the total length of the cable in the front side of the foot does not change and point $C$ does not move up or down. Additionally, since the cable goes through a pulley at point $\mathrm{C}$, it neither moves in any direction nor constrains the cable from motion.

The proposed design benefits from using two motors simultaneously. The motor torques can be combined to produce DP torques and subtracted to produce IE torques. Considering the fact that more power is required in the sagittal plane than the frontal plane during walking, this approach is more appropriate than employing a larger motor for DP and one smaller motor for IE. Additionally, it does not require any extra hardware other than the universal joint and the pulley at the ankle. 
The cable-driven design, besides the ability to control the ankle in two DOFs, can provide significant flexibility for managing the ankle-foot inertia. The motors and gear boxes can be located remotely and still provide the necessary characteristics and user preferences. The actuation system has the potential to move up or down on the pylon to accommodate the users' residual limb or to optimize the weight distribution and inertia characteristics.

\section{Selection and design of the active components}

For an average able-bodied human weighing $80 \mathrm{~kg}$, the energy consumption required at each step is $36 \mathrm{~J}$ (250 watts peak power) [14] and torque as high as $140 \mathrm{Nm}$ [21] during walking. These amounts are 35\% higher for an individual with transtibial prosthesis [14,22], and the mechanism is estimated to have $40 \%$ losses, resulting in an anticipated peak power consumption of 470 watts, an energy consumption of $68 \mathrm{~J}$, and a peak torque of $264 \mathrm{Nm}$ in the sagittal plane. Also, the device needs to generate $120 \mathrm{deg} / \mathrm{s}$ for the sagittal plane and $101^{\circ} / \mathrm{s}$ for IE, as estimated from the gait experiment described previously.

Considering these requirements, the prototype anklefoot mechanism uses two brushed DC motors and motor controllers that are capable of a continuous torque output of $0.275 \mathrm{Nm}$ at 10,000 RPM (producing 240 watts each for a total of 480 watts). They are connected to gear boxes with a 104:1 reduction gear ratio. Two 11.1 V LiPo batteries connected in series with an energy density of $572 \mathrm{~kJ} / \mathrm{kg}$ are estimated to provide energy for 5,800 steps. Even though the current prototype is designed as a proof of concept, it weighs $3 \mathrm{~kg}$ without the battery and socket which is the equivalent weight of the ankle-foot of a 52-kg person [21]. Considering the 104:1 gear ratio of the gear box and the $0.0127 \mathrm{~m}$ radius of the cable drum, the pulling force at the cables, $F_{\text {pull }}$, can be found as:

$$
F_{\text {pull }}=\frac{T}{0.0127} \times 104
$$

where $T$ is the instantaneous torque generated by the motor. The cable speed $\dot{L}$ is defined as:

$$
\dot{L}=\frac{\omega}{60 \times 104} \times 2 \pi \times 0.0127
$$

where $\omega$ is the instantaneous speed of the motor in RPM. At $0.275 \mathrm{Nm}$ of torque and 10,000 RPM, the generated pulling force is $2,252 \mathrm{~N}$ at $0.127 \mathrm{~m} / \mathrm{s}$. The moment arm in the sagittal plane, the distance in the $Y$-axis direction from the center of the universal joint to the cables in the back of the carbon fiber plate, is $57 \mathrm{~mm}$. The moment arm in the frontal plane, the distance in the $X$-axis from the center of the universal joint to the cables in the back of plate, is $28.4 \mathrm{~mm}$. DP and IE torques can be found as follows:

$$
\begin{aligned}
\tau_{\mathrm{DP}}= & \left(F_{\text {pull-left }}+F_{\text {pull-right }}\right) \times 0.057 \\
& \times \cos \left(\theta_{D P}\right) \\
\tau_{I E}= & \left(F_{\text {pull-left }}-F_{\text {pull-right }}\right) \times 0.0284 \times \cos \left(\theta_{I E}\right)
\end{aligned}
$$

where $\tau_{\mathrm{DP}}$ and $\tau_{\mathrm{IE}}$ are torques in DP and the IE, respectively, and $F_{\text {pull - left }}$ and $F_{\text {pull - right }}$ are the pulling forces from the left and right motors.

Due to the design geometry, when the foot deviates from the neutral position, the cables do not connect to the carbon fiber plate at a $90^{\circ}$ angle, resulting in losses of the generated torque. These losses are taken into the account by $\cos \left(\theta_{D P}\right)$ and $\cos \left(\theta_{I E}\right)$ terms in Equations 3 and 4 , respectively. At the maximum angular displacements observed in the gait experiment, the prototype shows a $7 \%$ loss in DP torque at $-21.6^{\circ}$ plantarflexion (maximum DP angle) and a $6 \%$ loss in IE at $19.7^{\circ}$ inversion (maximum IE angle). These nonlinear losses are small but should be considered during the control of the mechanism.

The angular velocities $\dot{\theta}_{D P}$ and $\dot{\theta}_{I E}$ are as follows:

$$
\dot{\theta}_{D P}=\frac{\dot{L}_{\text {Right }}+\dot{L}_{\text {Left }}}{2 \times 0.057} \times \frac{180}{\pi}
$$

and:

$$
\dot{\theta}_{I E}=\frac{\dot{L}_{\text {Right }}-\dot{L}_{\text {Left }}}{2 \times 0.0284} \times \frac{180}{\pi}
$$

where $\dot{L}_{\text {Left }}$ and $\dot{L}_{\text {Right }}$ are the cable velocities pulling from the left and right motors, respectively. The motors (at $0.275 \mathrm{Nm}$ torque and 10,000 RPM) are capable of generating $256 \mathrm{Nm}$ at $128^{\circ} / \mathrm{s}$ in DP and $64 \mathrm{Nm}$ at $256^{\circ} / \mathrm{s}$ in IE. These values are close to the estimated $264 \mathrm{Nm}$ of required torque in the sagittal plane based on the maximum torque required for walking [21] and the expected losses in the prosthesis and close to the angular velocities of $120 \%$ for DP and $101 \%$ s for IE measured in the gait experiment described previously.

\section{Selection and design of the passive component}

The shock-absorbing nylon rope stretches to provide shock absorption while being soft to the touch. Nylon ropes are strong and flexible which makes it ideal for the design of this prototype. The carbon fiber plate is $3.175 \mathrm{~mm}$ thick and is a fundamental component of the design. It has been reported that the average plantarflexion stiffness at $50 \% \mathrm{MVC}$ is $143 \mathrm{Nm} /{ }^{\circ}$ and is near constant from $30 \%$ to $80 \%$ MVC [23]; thus, the carbon fiber plate was designed to provide a stiffness comparable to these amounts in the sagittal plane. The carbon fiber plate acts as a spring connected in series between 
the cable and foot. The cable needs to be always in tension to assure the proper control over the foot, causing the carbon fiber plate to be always under a bending moment.

\section{Maximum force, torque, and range of motion}

A Kistler ${ }^{\oplus}$ Type 5233A force plate was used to measure the maximum amount of force produced by the anklefoot mechanism with a step input in plantarflexion. The mechanism's pylon was constrained in the $z$ direction with the intention to measure the torque at the ankle while DC motors were applying the stall torques. The device was capable of generating a lift force of $712 \mathrm{~N}$, which corresponds to $103.2 \mathrm{Nm}$ torque. The maxim IE torque can be calculated directly from the DP torque and the mechanism's geometry to an estimated $25.8 \mathrm{Nm}$.

In the current configuration, the maximum range of rotation recorded from the position encoders was $49^{\circ}$ in DP and $98^{\circ}$ in IE, due to the range of rotation of the cable drums. Both DP and IE ranges are within the set goals. Testing the ROM in IE, it was found that the IE motion becomes unstable at angles above $62^{\circ}$ (if forced by an external torque to this angle) similar to rolling of the ankle, which is an occurring injury among sport players and is in inversion [24]. In the prototype mechanism, ankle roll happens when either points A or B (Figure 3) crosses the $Y$-axis. At this configuration, the tension in the cables, which are applying a torque against the disturbance force, change the direction of the torque and makes the ankle unstable. The maximum expected rotation observed in IE during the gait tests using the camera system was $19.7^{\circ}$ and thus instability should not be a problem during reproduction of a straight walk or turn.

\section{Instrumentation and control}

\section{Ankle torque and angle feedback}

To actively regulate the ankle impedance, torque and angle feedbacks are required. The prototype is equipped with strain gauges mounted to the foot, which can be correlated to the external torques and will be used in our future work for impedance modulation of the prototype. Strain gauges are commonly used in load cells to measure the strains in structures due to external forces. The strain gauges change the resistance as they stretch or contract, and their change in resistance is correlated to the strain of the object they are attached to and consequentially the force or torque applied to the object. Strain gauges are typically wired in a Wheatstone bridge configuration using four strain gauges (some of the strain gauges can be replaced by resistors). The increase or decrease in resistance of two of the strain gauges (placed in opposite sides of the bridge) causes the output voltage of the bridge to increase or decrease, respectively. The opposite is also true for the other two strain gauges where the increase or decrease in resistance decreases or increases the output voltage of the bridge, respectively.

For estimating the torque in DP, four strain gauges were attached to the sole of the foot (Figure 4A). Two strain gauges $\mathrm{S}_{\mathrm{dp}} 2$ were placed behind the center of rotation of the ankle in the sagittal plane and were wired into opposite sides of the Wheatstone bridge (Figure 5A). Any ground reaction force at the hill caused a decrease in the voltage of the Wheatstone bridge, which could be correlated to the torque in the sagittal plane of the foot when the heel was interacting with the ground (e.g., heel strike). Two strain gauges $\mathrm{S}_{\mathrm{dp}} 1$ were located in front of the center of rotation of the ankle in the sagittal plane and wired in the opposite sides of the Wheatstone

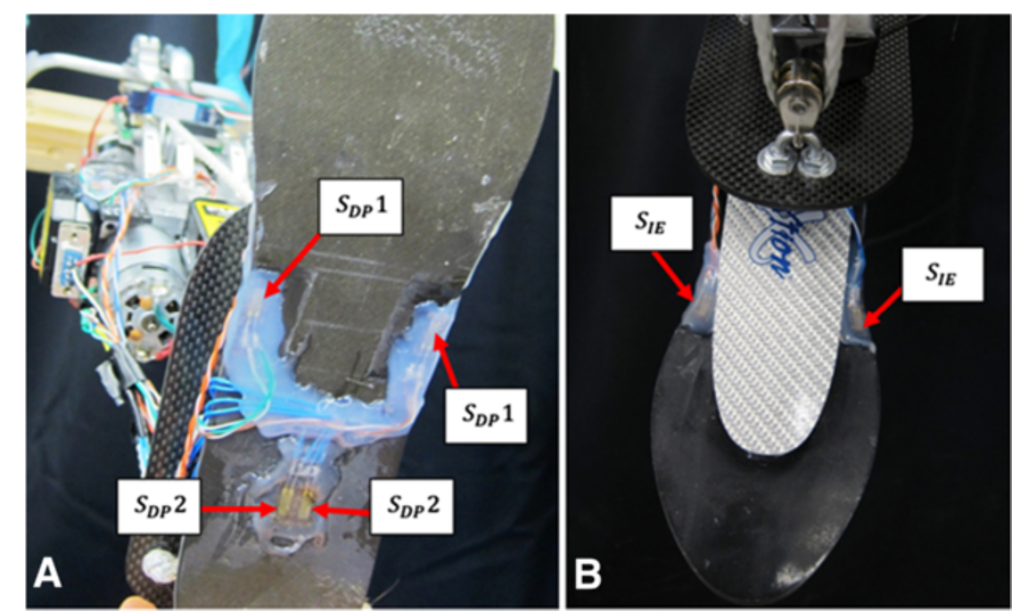

Figure 4 Strain gauge placement in the foot. (A) For the sagittal plane torque, two strain gauges are used for push-off torque estimation $\left(S_{D P} 1\right)$ and two strain gauges are used for heel-strike torque estimation $\left(S_{D P} 2\right)$. (B) For the frontal plane torque, two strain gauges are used for push-off torque estimation $\left(S_{\mid E} 1\right)$. 


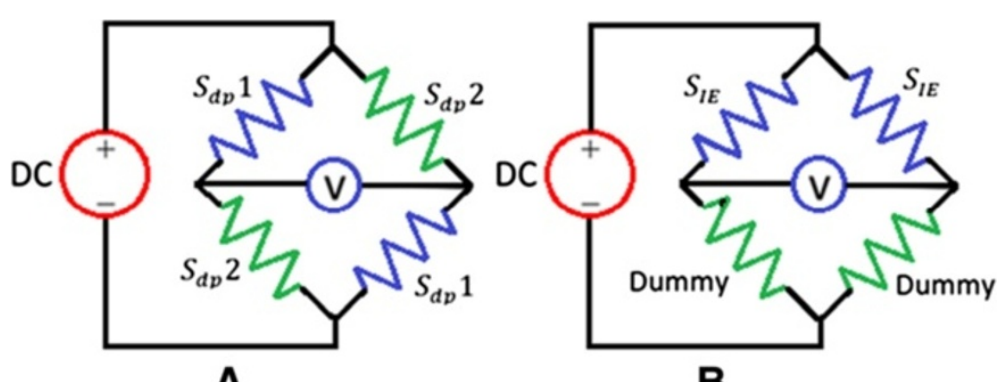

A

B

Figure $\mathbf{5}$ Wheatstone bridges. (A) Strain gauge placement in the Wheatstone bridge for the sagittal plane torque estimation. Two strain gauges are used for push-off torque estimation in the sagittal plane $\left(S_{D P} 1\right)$, and two strain gauges are used for heel-strike torque estimation in the sagittal plane $\left(S_{D P} 2\right)$. (B) Strain gauge placement in the Wheatstone bridge for the frontal plane torque estimation. Two strain gauges are used for push-off the frontal plane torque estimation $\left(\mathrm{S}_{\mathrm{IE}}\right)$.

bridge (Figure 5A). Any ground reaction force at the front of the foot caused an increase in the output voltage of the Wheatstone bridge, which could be correlated to the torque in the sagittal plane of the foot when it was contacting the ground (e.g., push-off). Note that when the foot was flat on the ground, the output from the strain gauges $\mathrm{S}_{\mathrm{dp}} 1$ canceled the output from the strain gauges $\mathrm{S}_{\mathrm{dp}} 2$; therefore, the resultant voltage could always be correlated to the net sagittal plane torque in the ankle.

For estimating the torque in IE, two strain gauges were attached to the top surface of the foot as shown in Figure 4B. The other two strain gauges were attached to an inert piece of carbon fiber but could also be replaced by two resistors with resistance identical to the strain gauges. The strain gauges were placed on the outside edges of the foot and were on the same side of the Wheatstone bridge (Figure 5B); hence, the difference in strains caused an increase or decrease in voltage at the bridge. The voltage could be correlated to the IE torque in the foot when the front of the foot was contacting the ground (e.g., push-off). This configuration made the bridge insensitive to the torque in the sagittal plane, since the strain gauges were in the opposite ends of the bridge. Therefore, if they both contracted or stretched by the same amount as it would happen in the presence of a DP torque, the output would not be affected. This feature was important since it was necessary to have the frontal plane torque estimation to be decoupled from the sagittal plane torque.

To correlate the strain gauge readings to the actual disturbance torques, a Kistler ${ }^{\oplus}$ Type $5233 \mathrm{~A}$ force plate was used to measure the external force applied during quasi-static loading tests. The tests consisted of loading the foot in different configurations and recording the applied force and the corresponding strain measurement. The tests were performed in 1 - plantarflexion by applying a load when the heel was in contact with the ground, 2 - dorsiflexion by applying a load when the forefoot was in contact with the ground, 3 - eversion by applying a load when the right edge of the forefoot was in contact with the ground, and 4 - inversion by applying a load when the left edge of the forefoot was in contact with the ground. From the external forces, the geometry of the foot, and the strain measurements, the applied torques were calculated. It is important to note that in the sagittal plane, the proportional factor between the external force and the strains measured at heel loading and forefoot loading were not the same, since the strain gauges were attached to two different areas of the foot. The proportional factors for the strain gauges at heel loading and forefoot loading were estimated at 1.41 and $19.52 \mathrm{Nm} /$ volt, respectively. As a result, different proportional factors needed to be used depending if the strain measurement was positive or negative. In the frontal plane, the proportional factors for inversion and eversion torques were closer (4.43 and $3.55 \mathrm{Nm} /$ volt, respectively), which was expected since the foot is near symmetrical about its sagittal plane.

\section{Position controller}

Presently, two optical quadrature encoders (200 pulses per revolution) provide position feedback to a proportional plus derivative (PD) controller to control the relative position of the foot with respect to the pylon. The overall control architecture of the ankle-foot mechanism can be seen in Figure 6. The block diagram of the position controller is shown in Figure 7.

The overall controller consists of two identical PD controllers to estimate the appropriate motor voltages. The input to the left motor controller is the sum of DP and IE angles, while the input to the right motor controller is the difference between the DP and IE angles. The desired angular displacements were the ankle angles measured during the gait experiment using the camera system that were stored in a lookup table to be reproduced by the ankle-foot mechanism for evaluation purposes as discussed in the following sections. 


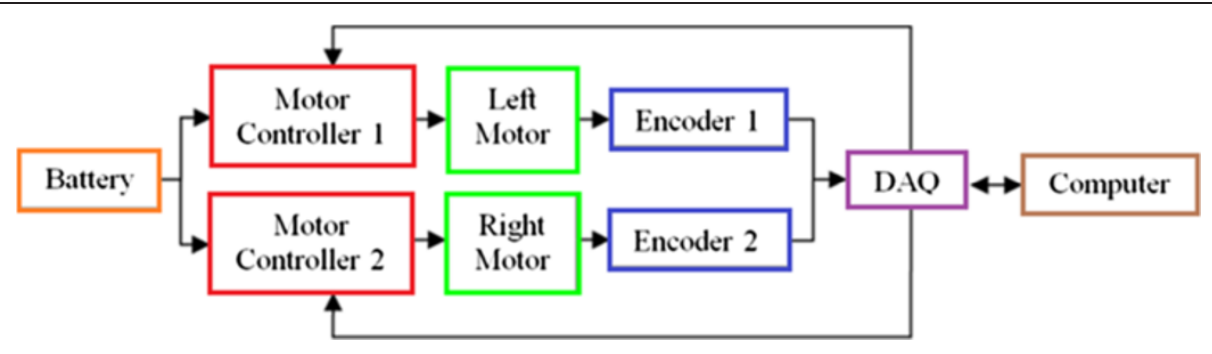

Figure 6 Overall control architecture of the ankle-foot mechanism.

\section{Evaluation of the ankle-foot mechanism \\ Tracking performance}

The aim of this evaluation study is to show that the mechanism is capable of reproducing the human ankle rotations during straight walk and turning. The input of the controller is the time history of a representative subject's ankle angles in DP and IE recorded with a camera system and stored in a lookup table. The output is the ankle angles calculated using the quadrature encoders.

The angular displacement of the ankle in DP and IE ( $\theta_{D P}$ and $\theta_{I E}$, respectively) can be calculated from the left and right quadrature encoders' feedback ( $\theta_{\text {Left }}$ and $\theta_{\text {Right }}$, respectively), where $K_{\mathrm{DP}}$ and $K_{\mathrm{IE}}$ are constant gains to define the ankle rotations as a function of the quadrature encoders' outputs and are based on the mechanism's geometry.

$$
\begin{aligned}
& \theta_{D \mathrm{P}}=K_{D P}\left(\frac{\theta_{\text {Left }}+\theta_{\text {Right }}}{2}\right) \\
& \theta_{I E}=K_{I E}\left(\theta_{\text {Right }}-\theta_{\text {Left }}\right)
\end{aligned}
$$

The input and output angles of the position controller can be seen in Figure 8 when the reference angle of the controller is updated at a rate of $125 \mathrm{~Hz}$. For ease of comparison, the output plots have a time shift to remove the $24 \mathrm{~ms}$ delay of the output. Also, all signals are filtered with a low-pass filter with a cut-off frequency of $5 \mathrm{~Hz}$ to remove frequency components from the input that are beyond the bandwidth of the motors currently installed, as well as sensor noises from the output signal.

Due to the physical characteristics of the mechanism, small angular differences between the left and right motors caused larger changes in the foot rotations, making the system more sensitive to disturbances and noise in the frontal plane compared to the sagittal plane. Note that the current prototype was developed as a proof of concept to validate the design kinematics and uses lowcost brushed DC motors and gear boxes with limited bandwidth, which are the cause of the observed time delay. Therefore, faster motors and sensors with lower noise levels will be used in future designs. Nevertheless, both plots indicate that the mechanism is capable of reproducing the ankle rotations of a human subject during step turn with desirable accuracy.

\section{Impedance test}

Mechanical impedance of a system is the relationship between the displacement input and its force outputs. Control strategies have been developed for impedance control of active prostheses [25,10], where higher level control identifies the gait cycle and the lower level control regulates the actuators impedance characteristics. Ankle-foot robots can be designed to show passive and active mechanical impedance similar to the human ankle. In a stationary position, the passive mechanical impedance is dependent only to the passive components of the device. If the passive mechanical impedance of the mechanism is similar to the human ankle mechanical impedance, it may allow the impedance controller to easily regulate the active mechanical impedance of the mechanism to the target impedance for each specific gait cycle.

An impedance estimation test was conducted to evaluate the passive impedance of the ankle-foot mechanism

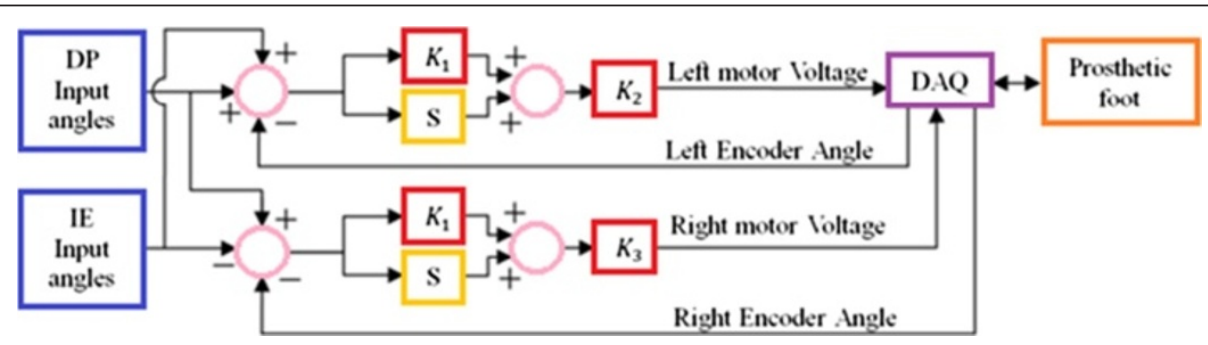

Figure 7 Block diagram of the ankle-foot mechanism position controller. 

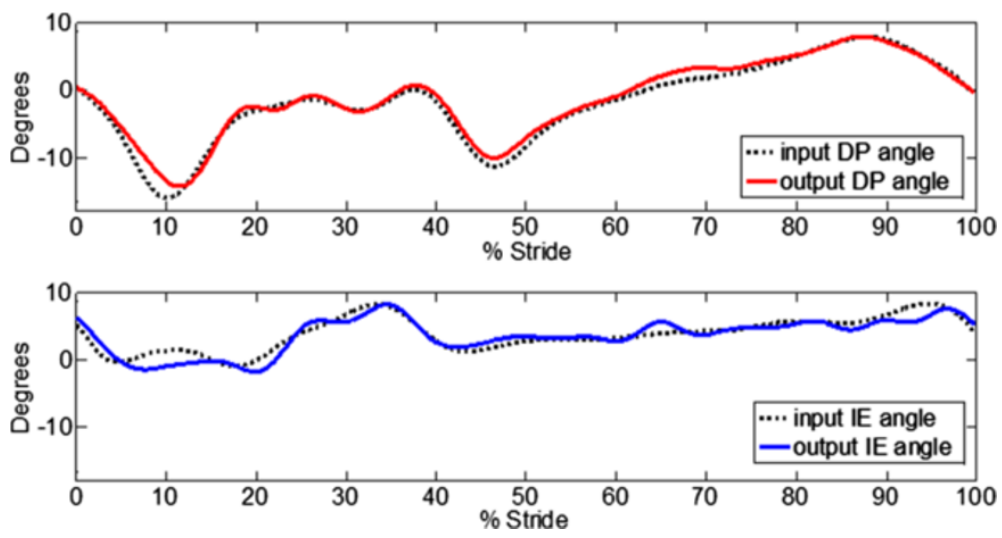

Figure 8 Input and compensated output for time delay $(24 \mathrm{~ms})$ of the ankle-foot mechanism. The input is the recorded right ankle rotations of a representative subject during swing and stance periods of the sidestep cutting at fast speed.

to compare it to the passive and active impedance of a human subject. The experiment was similar to the tests performed in [26,27] where the Anklebot, a multi-axis lower extremity therapeutic robot, was employed to apply pseudo-random torque perturbations to the ankle in DP and IE directions. The prototype mechanism attached to the Anklebot is shown in Figure 9, where the Anklebot and the ankle-foot mechanism were attached mechanically to each other. The passive mechanical impedance of the mechanism was measured with all the controllers of the mechanism turned off. For this test, the rubber foot shell was inserted in the same type of shoe used for human tests to ensure consistency in the experiments.

Anklebot provided the input torques to the ankle joint of the prototype mechanism $\left(\tau_{D P}\right.$ and $\left.\tau_{I E}\right)$ and also measured the resultant ankle displacements $\left(\theta_{D P}\right.$ and $\left.\theta_{I E}\right)$. A stochastic system identification method was used to estimate the mechanical impedance of the mechanism's ankle in DP and IE directions, $Z_{D P}(f)$ and $Z_{I E}(f)$, respectively.

$$
\begin{aligned}
& \tau_{D P}=Z_{D P}(f) \theta_{D P} \\
& \tau_{I E}=Z_{I E}(f) \theta_{I E}
\end{aligned}
$$

Sixty seconds of data were sampled at $200 \mathrm{~Hz}$ yielding 12,000 samples for both Anklebot and for the combined Anklebot and ankle-foot mechanism. Welch's averaged, modified periodogram method of spectral estimation, as implemented in MATLAB, was used to estimate onesided auto and cross-power spectral densities of the torque and angle sequences in the DP and IE directions. A periodic Hamming window with a length of 512 samples was used with an FFT length of 1,024 samples, yielding a spectral resolution of $0.195 \mathrm{~Hz}$. Power spectral density functions were estimated by averaging their values calculated from 45 data windows with $50 \%$ overlap (256 samples).

The foot and Anklebot's shoe share the same motion; therefore, the mechanical impedance of the prototype mechanism's ankle was obtained by subtracting the Anklebot mechanical impedance from the total estimated mechanical impedance as follows.

$$
Z_{\text {ankle }}=Z_{\text {ankle }+ \text { Anklebot }}-Z_{\text {Anklebot }}
$$

To compare the mechanical impedance of the anklefoot mechanism with the mechanical impedance of human ankle, experiments were performed on a human subject with relaxed muscles and with $10 \%$ of maximum voluntary contraction (MVC) of the lower leg muscles, which was maintained by monitoring the surface electromyography (EMG) of the tibialis anterior muscle of the subject, following the procedures described in [26]. Figure 10 shows the Bode plots of the mechanical impedances in the sagittal plane of the prototype mechanism, the human subject's ankle with relaxed muscles, and the human subject's ankle with 10\% MVC. The quasi-static stiffness magnitude of the prototype mechanism was $39.5 \mathrm{~dB}$ (94 Nm/radian) in the sagittal plane at below $1 \mathrm{~Hz}$ with relatively linear impedance and phase characteristics up to $5 \mathrm{~Hz}$ (the frequency range of interest [15]), with an average coherence value of 0.92 . The human subject showed similar stiffness in the sagittal plane for the cocontraction test and a lower quasi-static stiffness magnitude in the passive test when compared to the ankle mechanism. The frontal plane impedance magnitude (Figure 11) of the mechanism was between the active and passive stiffness of the human subject's ankle impedance with a value of $24.24 \mathrm{~dB}(16 \mathrm{Nm} /$ radian $)$ at below $1 \mathrm{~Hz}$. Considering that the magnitude of the ankle impedance is different among individuals, the results showed that it is feasible to design a mechanism with impedance 


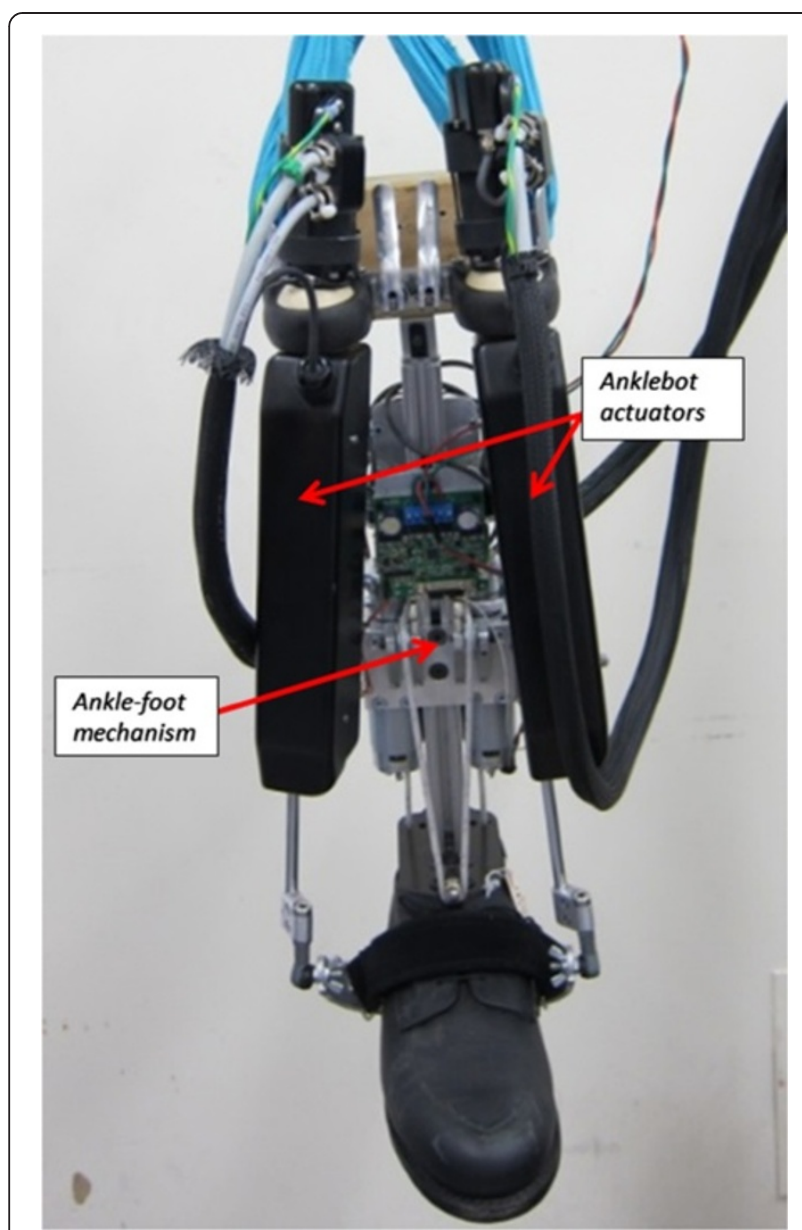

Figure 9 Anklebot attached to the ankle mechanism for estimation of the mechanical impedance of the ankle-foot mechanism.

characteristics close to the human ankle impedance, allowing for fine tuning of these values by an impedance controller that will be designed in the future.

\section{Results and discussion \\ Ankle rotation}

Ankle rotations during straight walk and step turn were measured using a camera system to study the ankle function in the frontal plane during the turn. The ankle rotations in the frontal plane during straight walk in both slow and fast speeds were close; however, there was a significant increase in the ankle's rotation in the IE direction during the step turn. The ankle angles in IE during the push-off increased from $2.8^{\circ}$ eversion during straight walk at low speed to $10.5^{\circ}$ inversion during the step turn at slow speed. These values were $2.5^{\circ}$ eversion and $14.3^{\circ}$ inversions during the step turn for straight walk and step turn, respectively. These changes were equivalent to $248 \%$ and $312 \%$ of the straight step average ROM in the frontal plane for the slow and fast speed tests, respectively. The changes observed in the angular displacement of the ankle in the frontal plane during step turn, when compared to straight walk, showed the importance of the IE rotation during turning.

\section{Mechanical characteristics of the ankle mechanism}

The prototype ankle-foot mechanism was designed to control the foot in two DOFs. The mechanism uses two identical motors to generate torques in the sagittal plane and in the frontal plane. When both motors rotate in the same direction, they generate DP motion at the foot. IE motion is also generated when the motors move in opposite directions. Sagittal plane torque requirements are larger than the frontal plane torque requirements, and the design benefits from its combined power from both motors to generate DP torques and difference in torques to generate IE torques, instead of using one large motor for DP and one smaller motor for IE. The cable design also allows for placement of the motors at different places on the pylon. This allows the design to be easily adjusted for amputees with different residual limb length or to optimize weight distribution. Additionally, the design may be significantly improved using Bowden cables, allowing the motors and gear boxes to be mechanically decoupled from the pylon and placed elsewhere. This will allow for great flexibility on the design and customization of the prostheses based on individual user needs, especially for amputees with long residual limbs. Also, having the flexibility of placing the motors and gearboxes, which carry the most of the weight of the mechanism, away from the distal end of tibia would allow for a lightweight prosthesis that in theory can result in more symmetric gait patterns.

The prototype ankle mechanism was designed to match the mechanical characteristics of the human ankle. This included power, range of motion, and weight. The maximum power consumption, based on the energy expenditure of humans and the expected losses in the mechanism, is 470 watts. The motors chosen for the ankle mechanism have a combined power of 480 watts. The motors are rated at $0.275 \mathrm{Nm}$ torque at 10,000 RPM, which results in $256 \mathrm{Nm}$ at $128^{\circ} / \mathrm{s}$ in DP and $64 \mathrm{Nm}$ at $256 \% \mathrm{~s}$ in IE. These values are similar to the estimated $264 \mathrm{Nm}$ of required torque in plantarflexion, and the angular velocities are close to 120 and $101^{\circ}$ s required for the sagittal plane and the frontal plane motion, respectively. Using a force plate, the maximum lifting force of the ankle mechanism was found to be $712 \mathrm{~N}$ in the sagittal plane which is the equivalent of the weight of a $73-\mathrm{kg}$ person.

Impedance controllers have been used in active prosthesis to control the position and torque generated by the device. An impedance controller requires both position feedback and force feedback of the interactive forces between the device and the environment. The 


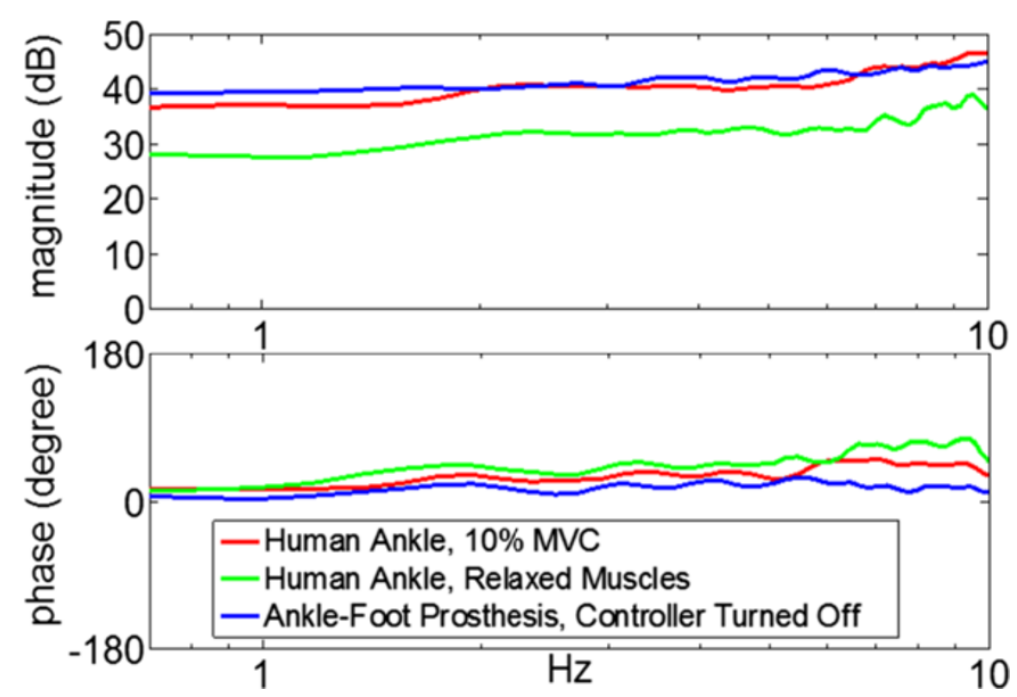

Figure 10 Bode plot of the ankle impedance in the sagittal plane. Impedance of the ankle-foot mechanism and a human subject with both relaxed muscles and 10\% MVC. The top plot is the magnitude and the bottom plot is the phase. The quasi-static impedance of the mechanism was above the human ankle impedance magnitude with 10\% MVC at low frequencies (around $1 \mathrm{~Hz}$ ).

ankle mechanism in its current form uses quadrature encoders at the cable drums for position feedback. This provides an estimate of the ankle angles but does not account for the nonlinearities of the device caused by the rotation of the ankle, or the deflection of the carbon fiber plate, and stretch of the nylon rope. This can be remedied by using the encoders to measure the rotation of the foot directly, instead of the cable drums' rotations. The torques generated from the mechanism can be measured using strain gauges at the foot, as described in this paper. However, the nonlinearities caused by the rotation of the foot also affect the generated torque, which need

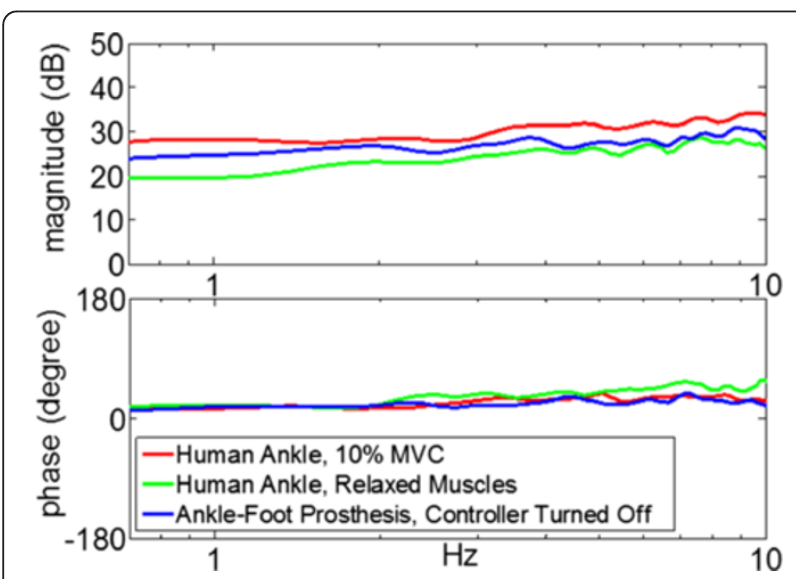

Figure 11 Bode plot of the magnitude and phase of the ankle impedance in the frontal plane. Impedance of the ankle-foot mechanism and a human subject with relaxed muscles and $10 \%$ MVC. The top plot is the magnitude, and the bottom plot is the phase. The quasi-static impedance of the mechanism was in between of the human ankle impedance magnitude with relaxed muscles and with 10\% MVC at low frequencies (around $1 \mathrm{~Hz}$ ). to be accounted in the controller design in the future. This will allow the use of ground reaction torques feedback and the angular displacements of the foot in the implementation of impedance controllers for the twoaxis ankle mechanism in both the sagittal plane and the frontal plane.

\section{Design evaluation}

Using a simple position controller, the recorded ankle rotations of a subject were reproduced by the prototype ankle mechanism. It was observed that the device was capable of tracking the human ankle motion in both the sagittal plane and the frontal plane. Since the mechanism was designed to use the difference in motor torques and angles to generate frontal plane torques and angular displacements, respectively, the design was found to be more sensitive to disturbances and noise in IE in comparison to DP.

The impedance estimation test showed that the quasistatic stiffness (below $1 \mathrm{~Hz}$ ) of the prototype mechanism was $39.5 \mathrm{~dB}(94 \mathrm{Nm} /$ radian$)$ in the sagittal plane with relatively linear impedance and phase characteristics up to $5 \mathrm{~Hz}$, which is the frequency of interest. The linearity of the system, shown as the average coherence of the estimated impedance, was 0.92 . These values were similar to the ankle stiffness of a human subject in the sagittal plane during the muscle co-contraction test and higher than the subjects' quasi-static stiffness with relaxed muscles. In the frontal plane, the mechanism impedance was between the active and passive stiffness of the human subject's ankle, with a stiffness of $24.24 \mathrm{~dB}(16 \mathrm{Nm} / \mathrm{ra}-$ dian). Considering the variation in ankle stiffness among individuals, these experiments show that the mechanism 
can be designed with a passive impedance similar to the human ankle. This will facilitate controlling the impedance of the mechanism through an impedance controller to the necessary values based on the gait requirements.

The current prototype was developed as a proof of concept to validate the design kinematics. It uses low-cost brushed DC motors and compatible gear boxes, resulting in low bandwidth of the motors and gear box, limited efficiency and power to weight ratio of the mechanism. Even so, the mechanism was capable of reproducing the ankle rotations of a human subject during step turn, adequate torques, and desirable mechanical impedance similar to a human ankle, while weighing $3 \mathrm{~kg}$. Therefore, using faster, brushless motors and sensors with lower noise levels in future designs will increase the efficiency, power, and bandwidth, while decreasing the device's weight.

\section{Conclusions}

In this paper, we described a novel two-axis, cabledriven ankle-foot mechanism. First, we described an experiment using a motion capturing camera system to study the human ankle during straight walk and turning, which showed that the inversion and eversion of the human ankle are significant during turning. This suggested that the design of ankle-foot mechanisms could be improved by controlling the degree of freedom of ankle mechanisms in the frontal plane. The proposed mechanism was described in detail, and its design parameters and considerations were presented. Moreover, a method for estimating the external torques applied to the ankle using strain gauges was presented, which is necessary in the development of impedance controllers. A simple position controller was implemented and used to demonstrate the capability of the device in tracking the same motion as the human ankle during a turning maneuver. It was shown that the prototype was capable of generating enough torque to lift a $73 \mathrm{~kg}$ person. An experiment was performed to explore the mechanism's passive mechanical impedance. The device showed passive mechanical impedance close to the impedance of a human subject, which prompts the feasibility of regulating the ankle-foot mechanism mechanical impedance using an impedance control. The presented mechanism was capable of providing key mechanical characteristics similar to the human ankle, including power, range of motion, and weight, while allowing instrumentation for the development of an impedance controller in both dorsiflexionplantarflexion and inversion-eversion.

\section{Abbreviations}

DOF: Degrees of freedom; DP: Dorsiflexion-plantarflexion;

EMG: Electromyography; IE: Inversion-eversion; LOPES: Lower extremity powered exoskeleton; MVC: Maximum voluntary contraction; ROM: Range of motion.

\section{Competing interests}

The authors declare that they have no competing interests.

\section{Authors' contributions}

All authors were fully involved in the study and preparation of the manuscript. All authors read and approved the final manuscript.

\section{Acknowledgement}

This material is based upon work supported by the National Science Foundation under CAREER Grant No. 1350154.

\section{Author details}

${ }^{1}$ Department of Mechanical Engineering-Engineering Mechanics, Michigan Technological University, Houghton, Michigan 49931, USA. ${ }^{2}$ Motion Analysis Laboratory, Department of Orthopedic Surgery, Mayo Clinic and Mayo Foundation, 201 W. Center St., Rochester, MN 55902, USA.

Received: 26 August 2014 Accepted: 15 October 2014

Published online: 02 November 2014

\section{References}

1. Veneman JF, Kruidhof R, Hekman EEG, Ekkelenkamp R, Asseldonk EHFV, Hvd K (2007) Design and evaluation of the LOPES exoskeleton robot for interactive gait rehabilitation. IEEE Trans Neural Syst Rehabil Eng 15(3):379-386

2. Glaister BC, Bernatz GC, Klute GK, Orendurff MS (2007) Video task analysis of turning during activities of daily living. Gait Posture 25(2):289-294

3. Glaister BC, Orendurff MS, Schoen JA, Bernatz GC, Klute GK (2008) Ground reaction forces and impulses during a transient turning maneuver. J Biomechanics 41(4):3090-3093

4. Ventura JD, Segal AD, Klute GK, Neptune RR (2011) Compensatory mechanisms of transtibial amputees during circular turning. Gait Posture 34:307-312

5. Orendurff MS, Segal AD, Berge JS, Flick KC, Spanier D, Klute GK (2006) The kinematics and kinetics of turning: limb asymmetries associated with walking a circular path. Gait Posture 23(1):106-111

6. Hase K, Stein RB (1999) Turning strategies during human walking. J Neurophysiol 81(6):2914-2922

7. Segal AD, Orendurff MS, Czerniecki JM, Schoen J, Klute GK (2011) Comparison of transtibial amputee and non-amputee biomechanics during a common turning task. Gait Posture 33(1):41-47. doi:10.1016/j. gaitpost.2010.09.021

8. Hogan N (1985) Impedance control: an approach to manipulation: part I-theory. J Dyn Sys Meas Control 107(1):1-7

9. Goldfarb M (2010) Powered robotic legs - leaping toward the future. Bioengineering, National Institute of Biomediacl Imaging and

10. Sup F, Bohara A, Goldfarb M (2008) Design and control of a powered transfemoral prosthesis. Int J Rob Res 27:263-273

11. Sup F, Varol HA, Mitchell J, Withrow TJ, Goldfarb M (2009) Preliminary evaluations of a self-contained anthropomorphic transfemoral prosthesis. IEEE ASME Transaction of Mechatronics 14(6):667-676

12. Sup F (2009) A powered self-contained knee and ankle prosthesis for near normal gait in transfemoral amputees. Vanderbilt University, Nashville, Tennessee

13. Hitt J, Merlo J, Johnston J, Holgate M, Boehler A, Hollander K, Sugar T (2010) Bionic running for unilateral transtibial military amputees. In: 27th Army Science Conference (ASC). Orlando, Florida

14. Hitt JK, Sugar TG, Holgate M, Bellman R (2010) An Active foot-ankle prosthesis with biomechanical energy regeneration. J Med Devices 4(1):011003

15. Au SK (2007) Powered ankle-foot prosthesis for the improvement of amputee walking economy. Massachusetts Institute of Technology, Cambridge, MA

16. Au S, Herr H (2008) Powered ankle-foot prosthesis. Robot Automation Mag 15(3):52-59

17. Au S, Weber J, Herr H (2009) Powered ankle-foot prosthesis improves walking metabolic economy. IEEE Trans Robot 25(1):51-66

18. Au SK, Herr H, Weber J, Martinez-Villalpando EC (2007) Powered ankle-foot prosthesis for the improvement of amputee ambulation. International Conference of the IEEE, Engineering in Medicine and Biology Society, Lyon.

19. Herr HM, Grabowski AM (2010) Powered ankle-foot prosthesis improves metabolic demand of unilateral transtibial amputees during walking. Amer Society of Biomechanics, Long Beach, CA. 
20. Bellman RD, Holgate MA, Sugar TG, Bellman RD, Holgate MA (2008) Sugar TG SPARKy 3: design of an active robotic ankle prosthesis with two actuated degrees of freedom using regenerative kinetics. In: Proceedings of the 2nd Biennial IEEE/RAS-EMBS International Conference on Biomedical Robotics and Biomechatronics. Scottsdale, AZ, USA, pp 511-516

21. Samuel K, Au JW, Hugh H (2007) Biomechanical design of a powered ankle-foot prosthesis. In: Paper presented at the International Conference on Rehabilitation Robotics, Noordwijk. The Netherlands

22. Rao SS, Boyd LA, Mulroy SJ, Bontrager EL, Gronley JK, Perry J (1998) Segment velocities in normal and transtibial amputees: prosthetic design implications. IEEE Trans Rehabil Eng 6(2):219-226

23. Sinkjaer T, Toft E, Andreassen S, Hornemann BC (1998) Muscle stiffness in human ankle dorsiflexors: intrinsic and reflex components. J Neurophysiol 60(3):1110-1121

24. Saripalli A, Wilson S (2005) Dynamic ankle stability and ankle orientation. In: Paper presented at the 7th Symp. Footwear Biomech. Conf, Cleveland, $\mathrm{OH}$

25. Eilenberg MF, Geyer H, Herr H (2010) Control of a powered ankle-foot prosthesis based on a neuromuscular model. IEEE Trans Neural Syst Rehabil Eng 18(2):164-173

26. Rastgaar M, Ho P, Lee H, Krebs HI, Hogan N (2010) Stochastic estimation of the multi-variable mechanical impedance of the human ankle with active muscles. In: ASME Dynamic Systems and Control Conference. Boston, MA

27. Rastgaar M, Ho P, Lee H, Krebs HI, Hogan N (2009) Stochastic estimation of multi-variable human ankle mechanical impedance. In: ASME Dynamic Systems and Control Conference. Hollywood, CA

doi:10.1186/s40638-014-0017-0

Cite this article as: Ficanha et al:: A two-axis cable-driven ankle-foot mechanism. Robotics and Biomimetics 2014 1:17.

\section{Submit your manuscript to a SpringerOpen ${ }^{\circ}$ journal and benefit from:}

- Convenient online submission

- Rigorous peer review

- Immediate publication on acceptance

- Open access: articles freely available online

- High visibility within the field

Retaining the copyright to your article

Submit your next manuscript at $\gg$ springeropen.com 